\title{
The effects of acute intermittent hypoxia on cardiovascular parameters in normotensive and chronic hypobaric hypoxia-induced hypertensive rabbits
}

\author{
Muhittin O. Yaman ${ }^{1}$, Ibrahim Guner ${ }^{1}$, Hafize Uzun ${ }^{2}$, Gulderen Sahin ${ }^{1}$ and Nermin \\ Yelmen $^{1}$ \\ ${ }^{1}$ Department of Physiology, Cerrahpasa Faculty of Medicine, Istanbul University, Istanbul, Turkey \\ ${ }^{2}$ Department of Biochemistry, Cerrahpasa Faculty of Medicine, Istanbul University, Istanbul, Turkey
}

\begin{abstract}
The effects of both chronic hypoxia and acute intermittent hypoxia on cardiovascular system are unclear. We designed this study to develop a rabbit model of hypertension by exposure to chronic hypobaric hypoxia $(\mathrm{CHH})$ and to investigate the effects of acute intermittent hypoxia on hypertensive rabbits. Present study was performed in 13 albino rabbits that divided into $\mathrm{CHH}$ and control groups. To develop hypertension, the rabbits were placed in a hypobaric chamber ( $390 \mathrm{mmHg} ; 22$ hours/day, 30 days). Afterwards, acute intermittent hypoxia protocol was applied (8\% $\mathrm{FIO}_{2}$ (Fraction of Inspired Oxygen) $1 \mathrm{~min}+5$ min normoxia, 20 cycles, 2 hours) to rabbits anesthetized with urethane and alpha-chloralose. Mean arterial pressure, heart rate and hematocrit values have been determined. Also asymmetric dimethylarginine (ADMA), endothelial nitric oxide synthase (eNOS), endothelin-1 (ET-1) and noradrenaline (NA) values have been analyzed in blood.

We developed a model of hypertension in rabbits via exposure to severe $\mathrm{CHH}$ and we believe that ADMA is an important parameter in the development and permanence of $\mathrm{CHH}$-induced hypertension. The main finding of this sudy was the depressor effect of acute intermittent hypoxia on blood pressure and heart rate in $\mathrm{CHH}$-induced hypertension model. Finally, we believe that acute intermittent hypoxia protocol may be applicable for prevention and treatment of hypertension if properly developed.
\end{abstract}

Key words: Hypertension - Noradrenaline - Acute intermittent hypoxia - ADMA — Endothelin-1

Abbrevations: ADMA, asymmetric dimethylarginine; $\mathrm{CHH}$, chronic hypobaric hypoxia; $\mathrm{DDAH}$, dimethylaminohydrolase; DP, diastolic pressure; eNOS, endothelial nitric oxide synthase; ET-1, endothelin-1; HR, hearth rate; HIF-1, hypoxia inducible factor-1; MAP, mean arterial pressure; NA, noradrenaline; NO, nitric oxide; SP, systolic pressure.

\section{Introduction}

Hypoxia is a common stress encountered under many conditions of health and disease. Recent studies have shown that hypoxia should have harmful or beneficial effects on

Correspondence to: Nermin Yelmen, Department of Physiology, Cerrahpasa Faculty of Medicine, Istanbul University, Istanbul, Turkey

E-mail: nermink@istanbul.edu.tr the organism related to its frequency, duration and severity. Acute severe hypoxia, chronic hypoxia and chronic intermittent hypoxia have been implicated as a risk factor for many cardiovascular and respiratory diseases such as pulmonary and systemic hypertension, myocardial infarction, stroke and central respiratory depression (Neubauer 2001; Prabhakar 2001; Guner et al. 2002, 2008; Row 2002; Barton et al. 2003; Calbet 2003; Yelmen et al. 2003, 2004, 2011; Prabhakar and Kumar 2004; Yelmen 2004; Foster et al. 2006). On the other hand, some studies suggested that chronic intermittent hy- 
poxia has beneficial effects especially on cardiovascular system like protection of heart against ischemia and prevention from arrhythmias (Meerson et al. 1987, 1989, 1993; Asemu et al. 2000; Zhong et al. 2002). In this situation, while chronic continuous hypoxia and intermittent hypoxia being held responsible for many cardiovascular diseases including systemic hypertension, different intermittent hypoxia protocols were reported to have beneficial effects on pulmonary and cardiovascular functions (Guner et al. 2007; Serebrovskaya et al. 2003, 2008). In addition, cardiovascular changes accompanying to chronic hypoxia and the regulatory mechanisms are not well acknowledged and the cardiovascular effects of acute intermittent hypoxia have not been studied at all.

For this reason, the first aim of our study was to investigate the cardiovascular changes mediated by chronic hypobaric hypoxia exposure and the underlying mechanisms. Secondly, investigating the effects of acute intermittent hypoxia on cardiovascular functions on control and chronic hypoxia exposed (hypertensive) rabbits.

\section{Materials and Methods}

In this trial, 13 adult albino rabbits weighing on average 3.5 $\pm 0.2 \mathrm{~kg}$ were used. Rabbits were divided into two groups: control (normotensive) $(n=6)$ and $\mathrm{CHH}$ (chronic hypobaric hypoxia exposed) $(n=7)$.

\section{Developing hypertension with chronic hypobaric hypoxia exposure}

The $\mathrm{CHH}$ group animals were kept at hypobaric chamber for $22 \mathrm{~h} /$ day, 30 days. The pressure of the chamber was stabilized at $390 \mathrm{mmHg}$ (corresponding to an altitude of $5550 \mathrm{~m}$ ) by an adjustable valve and a vacuum pump (Vaziri and Wang 1996).The temperature of the chamber was kept at $28^{\circ} \mathrm{C}$ with $12 \mathrm{~h}$ light and dark cycles. Hypobaric chamber was opened in the same hour every day and daily cares of the rabbits were performed.

The control group rabbits stayed in the same environment as the $\mathrm{CHH}$ rabbits with access to food and water ad libitum, except that they breathed normal room air. Before placing the rabbits in the chamber and after 30 days of chronic hypobaric hypoxia exposure, blood samples were collected from auricular veins of the animals and hematocrit values were determined.

\section{Anesthesia and surgical technique}

Rabbits were anesthetized with urethane (400 mg/kg i.v.; Sigma) and alpha-chloralose (40 mg/kg i.v.; Sigma). The level of anesthesia was determined that animals had reached a surgical plane of anesthesia when they became flaccid and no longer exhibited either an eye-blink or limb withdrawal. Tracheotomy was performed and the tracheal cannula connected to an inspiratory-expiratory valve was inserted into the trachea for hypoxic gas mixture breathing. The right femoral arterial catheter was used to collect arterial blood samples. The right femoral vein was cannulated for administration of both supplemental anesthetic and saline solution when necessary. All rabbits were given heparin $(500 \mathrm{U} / \mathrm{kg}$ i.v.) in order to prevent thrombosis which could occur before acute experiments. Animals were sacrificed by i.v. injection of an over dose of sodium pentobarbital $(500 \mathrm{mg} / \mathrm{kg})$ that caused rapid and irreversible cardiac arrest.

\section{Recordings}

For the recording of blood pressure and heart rate (HR), right femoral arterial cannula was connected to a Quad Bridge Amplifier (MLT 0380/D, ADInstruments) by means of Reusable BP Transducer (MLT 0380/D, ADInstruments). Mean arterial pressure (MAP), diastolic pressure (DP) and systolic pressure (SP) was calculated and recorded via Chart 5 software (ADInstruments).

\section{Acute experiment phases}

\section{Air phase}

Animals in the both groups were allowed to breathe room air (normoxic gas mixture) for 10 minutes to record the beginning values of blood pressure and heart rate values.

\section{Acute intermittent hypoxia phase}

Animals in both groups were initially allowed to breathe hypoxic gas mixture $\left(8 \% \mathrm{O}_{2}-92 \% \mathrm{~N}_{2}\right)$ for $1 \mathrm{~min}$. We subjected animals to 20 cycles of acute intermittent hypoxia for 60 seconds every 5 minutes for duration of 2 hours (Tamisier et al. 2005). The effect of acute intermittent hypoxia on blood pressure and heart rate were investigated.

Arterial blood samples were collected before and after acute intermittent hypoxia phase from both groups of animals for determination of biochemical parameters.

\section{Determination of biochemical parameters}

The blood from right femoral artery was collected into dry and heparinized tubes for the determination of biochemical parameters. After the blood samples were centrifuged for $5 \mathrm{~min}$ at $1000 \times g$ at $4^{\circ} \mathrm{C}$, plasma were stored at $-80^{\circ} \mathrm{C}$ until analysis. All parameters were analyzed in all samples together in a single batch; after we had finished our protocol (control and $\mathrm{CHH}$ samples were analyzed in the same batch). 
Assay of endothelin-1 (ET-1) concentrations

Plasma endothelin-1 levels were measured in duplicate aliquots, using enzyme-linked immunosorbent assay (ELISA) according to the manufacturer's instructions (USCN Life Science Inc. Wuhan China, cat.no: E0482Rb). The coefficients of intra- and interassay variations were $<10 \%$.

Assay of asymmetric dimethylarginine (ADMA) concentrations

Plasma ADMA levels were measured by enzyme immunoassay (EIA) using ADMA kit (USCN Life Science Inc. Wuhan China, cat.no: E1301Rb). The coefficients of intraand interassay variations were $<10 \%$.

\section{Assay of noradrenaline concentrations}

Plasma noradrenaline (NA) levels were measured in duplicate aliquots, using ELISA according to the manufacturer's instructions (USCN Life Science Inc. Wuhan China, cat.no: E0907Rb). The coefficients of intra- and interassay variations were $<10 \%$.

\section{Assay of endothelial nitric oxide synthase (eNOS) concentrations}

Plasma eNOS levels were measured in duplicate aliquots, using enzyme-linked ELISA according to the manufacturer's instructions (USCN Life Science Inc. Wuhan China, cat.no: $\mathrm{E} 0868 \mathrm{Rb})$. The coefficients of intra- and interassay variations were $<10 \%$.

\section{Statistical analysis}

SPSS statistical analysis program was used for the evaluation of the findings of this study. Statistical significance of the differences between experimental phases for control and $\mathrm{CHH}$ groups was determined by Wilcoxon Signed Rank Test. Mann-Whitney U test was used to determine statistical significance between the control and $\mathrm{CHH}$ groups. In all phases, $p<0.05$ was considered statistically significant.

\section{Results}

\section{The effects of chronic hypobaric hypoxia}

\section{Effects on cardiovascular parameters}

MAP, SP and DP values of the $\mathrm{CHH}$ group were found to be significantly higher $(p<0.01, p<0.05, p<0.01$, respectively) than that of control group (Table 1 ). HR values of both groups had no statisticaly significant changes.

\section{Effects on hematocrit}

Comparing the hematocrit values of $\mathrm{CHH}$ group of animals before $(40 \%)$ and after (55\%) chronic hypobaric hypoxia exposure, a significant increase $(p<0.05)$ has been determined (individual change). When hematocrit values of $\mathrm{CHH}$ group of animals (55\%) compared to control group animals hematocrit values (39\%), the increase in $\mathrm{CHH}$ group animals values were statisticaly significant $(p<0.05)$.

\section{Effects on NA, ET-1, ADMA and eNOS}

When plasma NA, ET-1, ADMA and eNOS values of $\mathrm{CHH}$ group animals were compared to control group animals, we found that only the ADMA levels $(p<0.01)$ of $\mathrm{CHH}$ group were significantly increased (Table 2 ). Other parameters tend to increase but had no statistical importance.

\section{The effects of acute intermittent hypoxia on control and CHH groups}

\section{Effects on cardiovascular parameters}

The cardiovascular responses were observed after 2 hours of acute intermittent hypoxia exposure ( $1 \mathrm{~min}$. hypoxia $\left(8 \% \mathrm{O}_{2}\right.$ $-92 \% \mathrm{~N}_{2}$ ), 5 min normoxia (room air), repeated 20 times) to both control and $\mathrm{CHH}$ group rabbits. Comparing the MAP, SP, DP, HR values of the control group animals before and after the acute intermittent hypoxia exposure, we found that

Table 1. Cardiovascular parameters in control and $\mathrm{CHH}$ group of rabbits treated with hypoxia during the experimental phases

\begin{tabular}{lllccc}
\hline \multirow{2}{*}{ Group } & $\begin{array}{l}\text { Experimental } \\
\text { phases }\end{array}$ & $\begin{array}{c}\text { MAP } \\
(\mathrm{mmHg})\end{array}$ & $\begin{array}{c}\text { SP } \\
(\mathrm{mmHg})\end{array}$ & $\begin{array}{c}\mathrm{DP} \\
(\mathrm{mmHg})\end{array}$ & $\begin{array}{c}\mathrm{HR} \\
\left(\mathrm{min}^{-1}\right)\end{array}$ \\
\hline \multirow{2}{*}{ Control } & Air & $72.2 \pm 4.0$ & $83.8 \pm 4.8$ & $61.1 \pm 3.5$ & $274.9 \pm 4.9$ \\
& AIH & $61.1 \pm 5.1^{*}$ & $71.5 \pm 7.3^{*}$ & $50.8 \pm 3.2^{*}$ & $200.3 \pm 17.5^{*}$ \\
\cline { 2 - 6 } CHH & Air & $92.6 \pm 2.0^{\# \#}$ & $103.7 \pm 3.2^{\#}$ & $82.5 \pm 1.9^{\# \#}$ & $258.0 \pm 9.1$ \\
& AIH & $73.9 \pm 3.7^{*}$ & $84.4 \pm 3.2^{*}$ & $62.5 \pm 5.3^{*}$ & $205.8 \pm 12.5^{*}$ \\
\hline
\end{tabular}

Values are means \pm SE. CHH, animals treated with chronic hypobaric hypoxia; AIH, acute intemittent hypoxia; MAP, mean arterial pressure; SP, systolic pressue; DP, diastolic pressure; HR, heart rate; $n$, the animal number in each group; ${ }^{\star} p<0.05$ indicates statistical significance of the changes before and after acute intermittent hypoxia phase in both control and CHH groups; ${ }^{\#} p<0.05,{ }^{\# \#} p<0.01$ indicate statistical significance of the means of the $\mathrm{CHH}$ group when compared with the control group. 
Table 2. Plasma NA, endothelin-1, ADMA and eNOS values in each group of rabbits during the experimental phases

\begin{tabular}{llccccc}
\hline Group & $\begin{array}{l}\text { Experimental } \\
\text { phases }\end{array}$ & $\begin{array}{c}\text { NA } \\
(\mathrm{pg} / \mathrm{ml})\end{array}$ & $\begin{array}{c}\text { Endothelin-1 } \\
(\mathrm{pg} / \mathrm{ml})\end{array}$ & $\begin{array}{c}\text { ADMA } \\
(\mathrm{nmol} / \mathrm{ml})\end{array}$ & $\begin{array}{c}\text { eNOS } \\
(\mathrm{pg} / \mathrm{ml})\end{array}$ & $n$ \\
\hline \multirow{2}{*}{ Control } & Air & $110.7 \pm 6.1$ & $17.9 \pm 7.6$ & $10.8 \pm 1.6$ & $1.1 \pm 0.5$ & 6 \\
& AIH & $117.5 \pm 1.7$ & $19.8 \pm 5.9$ & $16.1 \pm 0.4^{*}$ & $0.6 \pm 0.2^{*}$ & 7 \\
\cline { 2 - 7 } CHH & Air & $123.1 \pm 7.2$ & $34.3 \pm 9.5$ & $17.9 \pm 0.6^{\# \#}$ & $1.4 \pm 0.1$ & $7.6 \pm 0.1^{*}$ \\
\hline
\end{tabular}

Values are means \pm SE. CHH, animals treated with chronic hypobaric hypoxia AIH, acute intermittent hypoxia; NA, noradrenaline; ADMA, asymetric dimethyl arginine; eNOS, endothelial nitric oxide synthase; $\mathrm{n}$, the animal number in each group; ${ }^{\star} p<0.05$ indicates statistical significance of the changes after acute intermittent hypoxia phase in both control and CHH groups; ${ }^{\# \#} p<0.01$ indicates statistical significance of the air phase means of the $\mathrm{CHH}$ group when compared to the control group.

all the values significantly decreased $(p<0.05)$. Similar to control group animals, we found acute intermittent hypoxia exposure caused a significant decrease in MAP, SP, DP, HR values of the $\mathrm{CHH}$ group animals $(p<0.05)$ (Table 1$)$. When the percentage changes of the both group's responses to the acute intermittent hypoxia exposure compared, no significant difference were found (Fig. 1).

\section{Effects on NA, ET-1, ADMA and eNOS}

Comparing the basal plasma levels of NA, ET-1, ADMA and eNOS values of the control group animals with the values after the acute intermittent hypoxia exposure, we found that ADMA level significantly increased $(p<0.05)$ but eNOS level $(p<0.05)$ significantly decreased. The changes of NA and ET-1 levels had no statistical importance (Table 2).

When the basal plasma levels of NA, ET-1, ADMA and eNOS of the $\mathrm{CHH}$ group animals compared with the values after the acute intermittent hypoxia exposure, we found that ET- 1 and eNOS levels significantly decreased $(p<0.05)$ but

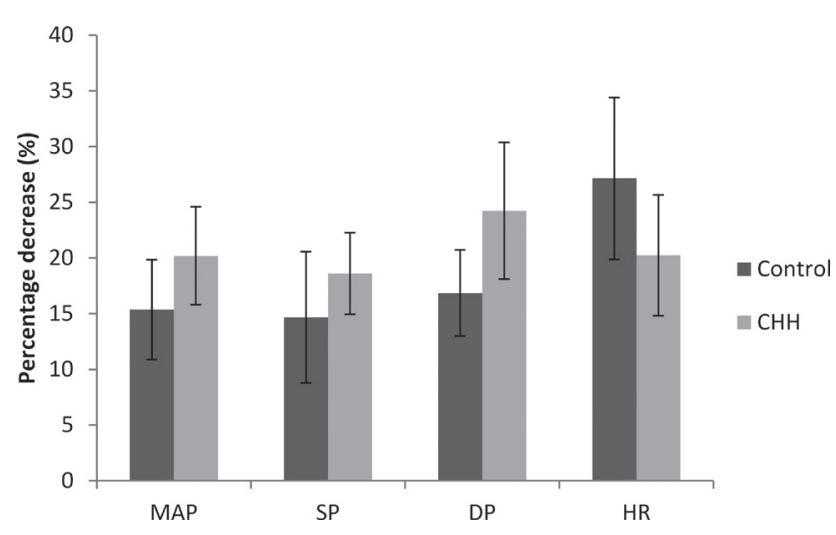

Figure 1. Comparison of the percentage changes of the decreased cardiovascular parameters after acute intermittent hypoxia exposure in control and chronic hypobaric hypoxia $(\mathrm{CHH})$ groups. MAP, mean arterial pressure; SP, systolic pressure; DP, diastolic pressure; HR, heart rate. Values are percentage means \pm SE.
NA and ADMA levels had no significant changes (Table 2). When we evaluated the percentage changes of plasma eNOS levels between $\mathrm{CHH}$ and control group animals, we determined that the percentage change of $\mathrm{CHH}$ group animals' plasma eNOS levels was significantly higher than control group animals' plasma eNOS \% change.

\section{Discussion}

In our study we have observed significant increases in MAP, SP and DP values of the rabbits exposed to chronic hypobaric hypoxia (CHH group) when compared to control group animals. Nearly $20 \mathrm{mmHg}$ of increase in MAP convinced us that developing hypertension with hypobaric hypoxia exposure is also possible for rabbits along with rats since we have not found any publication about a model for rabbits in literature (Vaziri and Wang 1996). A rabbit model is of great importance since rabbits are widely used as a model for human diseases, because of its size, which permits non-lethal monitoring of physiological changes and similar disease characteristics.

The effect of chronic hypoxia on blood pressure may vary due to its severity and duration. While chronic hypoxia exposures matching to very high altitudes (3500-5500 m) increases blood pressure (Hansen and Sander 2003), moderate hypoxia levels (1500-3000 m) tend to decrease blood pressure. Although we have shown that the severe hypoxia protocol we performed developed hypertension, the underlying mechanisms are not well known. We know that exposure to hypoxia exerts many adaptation reactions on organisms and hypoxia inducible factor-1 (HIF-1) plays a key role in regulation of these reactions (Semenza 2000). It is well acknowledged that chronic hypoxia increases hematocrit levels by increasing erythrocyte quantity via HIF-1 regulated gene activation. Increased hematocrit levels also observed in our study. Chronic hypoxia-mediated blood pressure elevation may be relevant to increased erythrocyte, blood volume and viscosity levels. But Vaziri and Wang showed that chronic 
hypoxia elevates blood pressure independent of hematocrit levels by performing phlebotomy to the rats (Vaziri and Wang 1996). We have not performed phlebotomy but we used the same protocol. Therefore, increased hematocrit levels may not be responsible for the elevation of blood pressure in our study. On the other hand, blood pressure elevation effect of chronic hypoxia may be related to sympathetic activity and active substances that affect vascular tone. For this reason, we determined the plasma levels of NA as a sympathetic activity marker, ET-1 as an important vasoconstrictor substance, eNOS as the responsible enzyme for NO synthesis in vascular endothelia and ADMA as an endogenous NOS inhibitor. We have observed that only the ADMA levels significantly increased in $\mathrm{CHH}$ group but NA, ET-1 and eNOS levels were not changed when compared to control group of animals. Former studies reported that the effect of hypoxia on sympathetic activity is associated with the severity and duration (Johnson et al. 1983). On the other hand, NO suggested to have influence on NA secretion from neuronal endings by some researchers. Yamamoto et al. suggested that NO increases the release of NA from the neuronal endings (Yamamoto et al. 1993). In contrast some researchers asserted that NO secreted by vascular endothelia is inhibiting NA secretion from sympathetic endings (Cohen and Weisbrod 1988; Greenberg et al. 1989). In this case, besides severity and duration of hypoxia, $\mathrm{NO}$ - one of the important variables in response to hypoxia may be effective on the sympathetic nerves that innervate blood vessels and thus on the level of plasma NA. We have observed increased plasma NA levels in CHH group animals compared to control group animals but the increase was statistically insignificant. Increased ADMA levels in CHH group animals may be held responsible from that change by inhibiting NO synthesis and therefore suppressing NA secretion (Yamamoto et al. 1993). The importance of NO on the regulation and sustainability of arterial blood pressure is well known (Moncada and Higgs 1995). We aimed to determine the changes in ADMA level which is an endogenous NOS inhibitor and the relation between ADMA and chronic hypoxia-mediated hypertension in our study. Recent studies suggest a direct relationship between ADMA and hypertension, and ADMA levels found elevated in hypertension parallel to our findings (Surdacki et al. 1999). But we did not find any publications about ADMA levels of chronic hypoxia-mediated hypertensive rabbits in literature. We have determined a slight increase in eNOS concentrations of chronic hypobaric hypoxia-exposed rabbits when compared to control group animals but the changes were not significant. The trend of increase in eNOS levels may indicate decreased basal NO production (Barua et al. 2001). Another important substance effecting vascular tone is ET-1. We have observed a non-significant increase in ET-1 levels of CHH group animals when compared to control group animals. Thus Vaziri and Wang stated that plasma ET-1 levels raised 3-fold during the second day and reached to a peak than started decreasing towards the basal level after seventh day of chronic hypobaric hypoxia exposure but never reached the initial basal plasma levels (Vaziri and Wang 1996). On the other hand several studies pointed out that chronic hypoxia-mediated hypertension cannot be prevented by ET-1 receptor antagonists (Ni et al. 1998) and also sustainability of hypoxia-mediated systemic arterial hypertension is not related to ET-1 or ET-1 receptors. Response to severe chronic hypoxic stress is related to downregulation of ET-A receptors response to ET-1 (Barbier 2007). As a result, increased ADMA levels may be an important determinant in development and sustainability of chronic hypobaric hypoxia-mediated hypertension. The changes in ET-1 levels and the effects of sympathetic activity (noradrenaline) should not be ignored but it is obvious that plasma concentration analyses of those parameters are not good indicators. Recording sympathetic neural activity would be more accurate while examining sympathetic activity. We are planning to record sympathetic neural activity in our future studies.

Recent studies suggest that different intermittent hypoxia protocols can be applied for therapeutic purposes (asthma, hypertension, cardiac arrhythmias (Serebrovskaya et al. 2003, 2008). These studies are mostly chronic studies of hypoxia matching to $4000-5000 \mathrm{~m}$ altitudes and carried for 3-12 weeks, 1 hour or more in a day, except the study of Altay et al. (2014) which concentrated on the effects of acute intermittent hypoxia on the cerebral microcirculatory inflammation and NO relationship. But the cardiovascular effects of acute intermittent hypoxia are not studied indepth. For this reason we have investigated the potential beneficial effects of acute intermittent hypoxia on the blood pressure and heart rate of rabbits and the mechanism of the effect. In our study; after 2 hours of intermittent hypoxia exposure applied to both control and $\mathrm{CHH}$ group rabbits, MAP, SP, DP and HR levels of both groups were decreased. Previously, a chronic intermittent hypoxia protocol applied for $30 \mathrm{~min} / \mathrm{d}, 10$ days has been shown to reduce blood pressure in hypertensive patients (Serebrovskaya et al. 2008). As is known, hypoxia is an important stress to the tissues and the organism. But repeated encounters with hypoxic stress occur because of the hypoxia and reoxygenation phases in intermittent hypoxia. The emergence of the positive effects of intermittent hypoxia has been hypothesized to rely on the basis of "a stress provides resistance to another" (Neubauer 2001). However, anti-hypertensive effect of acute intermittent hypoxia and the mechanism is unknown. The intermittent hypoxia protocol applied in our study is an acute protocol which consists of 20 hypoxia-reoxygenation cycles in 2 hours. The rabbits encounter repeated hypoxic stress like the chronic intermittent hypoxic protocols applied to the hypertensive patients which Serebrovskaya et al. stated 
but in a shorter period. We believe the acute intermittent hypoxia might effect in a similar manner.

It is well known that hypoxia is a key trigger for increased erythropoiesis. But intensity, frequency and duration of intermittent hypoxic cycles are likely the major determinants of erythropoesis and hematocrit (Rodriguez et al. 2000). Whether acute hypoxic exposure triggers erythropoietin (EPO) production, rising of hematocrit levels takes several days (West 2004). Therefore plasma hematocrit values are not expected to change after acute intermittent hypoxia exposure. For this reason, we have determined plasma NA levels with the thought in mind that our intermittent hypoxia procedure may be effecting blood pressure by sympathetic activity. But no significant changes in both groups NA levels were observed after acute intermittent hypoxia exposure. Dick et al. (2006) showed that acute intermittent hypoxia exposure $\left(8 \% \mathrm{O}_{2}-92 \% \mathrm{~N}_{2}, 10\right.$ times, 45 s hypoxia with $5 \mathrm{~min}$ intervals) increased sympathetic neuronal activity while blood pressure was not effected. But in our study, both group of animals' blood pressure dropped while plasma NA levels were not significantly changed. On the other hand, the acute intermittent hypoxia protocol we applied led to a decrease in heart rate. This finding supports the possible influence of increased parasympathetic activity in reduction of blood pressure mediated by acute intermittent hypoxia. Indeed, hypoxia has been proposed to increase the parasympathetic activity during acclimatization to high altitudes (Bernardi et al. 2001). Perhaps because of the hypoxic phases repeated during intermittent hypoxia may have developed such an adaptive response. Regarding the duration, severity and number of repetitions of intermittent hypoxia, sympathetic or parasympathetic influence may be dominant. While we have not measured the parasympathetic activity directly, we belive that the significant reduction in heart rate may be an indication of parasympathetic activity being more dominant for the hypoxia protocol we applied in our study. We aim to examine autonomic activity via power spectral analyses of heart rate in future studies to clarify the topic.

Considering the significant decrease in plasma eNOS levels and significant increase in plasma ADMA levels of control group animals, the decrease in eNOS concentrations may be due to increased NO levels or increased ADMA levels of the rabbits. We did not find any publication examined ADMAeNOS relation in acute intermittent hypoxia exposures. But the negative feedback relationship between eNOS concentrations and NO concentrations have been emphasized (Barua et al. 2001; Kim et al. 2006). Barua et al. stated that eNOS protein expression had a negative correlation with eNOS activity and $\mathrm{NO}$ concentrations in an in vitro smoking model and smoking was associated with decreased NO production while the plasma NO levels of the subjects were not significantly different. On the contrary, plasma ADMA concentrations found decreased while L-arginine concentrations and L-arginine/ADMA ratio found increased in high risk atherosclerotic group of smoking patients (Eid et al. 2003). Eventhough Antoniades et al. (2009) demonstrated that the increase in plasma ADMA concentrations were closely associated with decreased vascular NO bioavailability (Eid et al. 2003), Kim and co-workers found marginal decrease in eNOS protein expressions in preeclemptic patients which have decreased plasma L-arginine and L-arginine/ADMA levels rather than plasma ADMA concentrations (Kim et al. 2006). Just as the changes in our hypertensive group of rabbits which had no significant ADMA concentration changes but significantly decreased plasma eNOS levels. For better understanding of the difference in ADMA levels after acute intermittent hypoxia exposure between two groups, it may be possible to think that plasma ADMA levels were already elevated in $\mathrm{CHH}$ group animals and plasma DDAH (ADMA scavenging enzyme) levels may already be elevated, or an adaptation of excretion of ADMA from kidneys may be developed. Those may be the possible reasons for ADMA levels were not elevated further after acute intermittent hypoxia exposure in $\mathrm{CHH}$ group. We also determined that cardiovascular parameters were decreased in parallel with eNOS levels of both groups after acute intermittent hypoxia exposure. When we evaluated the percentage changes of plasma eNOS levels we have seen that the decrease in $\mathrm{CHH}$ group was higher. In this situation, we can say that acute intermittent hypoxia decreased cardiovascular parameters in proportion to plasma eNOS levels. In light of these findings, it is clear that further studies are needed to understand the effects of acute intermittent hypoxia on cardiovascular system. While we planned our study we were aware that plasma NO concentrations would not be a reliable measure for determining endothelium-derived NO but lacking the determination of L-arginine and L-arginine/ADMA ratios can be seen as a limitation of our study.

We have not observed significant changes in plasma ET-1 levels of control group rabbits in acute intermittent hypoxia exposure. But ET-1 levels of hypertensive rabbits were significantly decreased. A decrease in a vasoconstrictor substance as ET-1 is supportive finding with the decreased blood pressure.

\section{Conclusion}

We developed a model of hypertension in rabbits via exposure to severe chronic hypobaric hypoxia and we believe that ADMA is an important parameter in the development and sustainability of chronic hypobaric hypoxia-induced hypertension. The main finding of this study was the depressor effect of acute intermittent hypoxia on blood pressure and heart rate in chronic hypobaric hypoxia-induced hypertension model. Finally, we believe that acute intermit- 
tent hypoxia protocol may be applicable for treatment of hypertensive and high heart rated patients if proper methods will be developed.

Acknowledgments. All experimental protocols were performed in accordance with the National Institutes of Health guidelines and the approval of the Istanbul University Animal Care and Use Ethics Committee. This work was supported by the Research Fund of Istanbul University, Project No:3833.

The author's have no conflicts of interest or financial ties to disclose.

\section{References}

Altay T., Gonzales E. R., Rark T. S., Gidday J. M. (2004): Cerebrovascular inflammation after brief episodic hypoxia: modulation by neuronal and endothelial nitric oxide synthase. J. Appl. Physiol. 96, 1223-1230 http://dx.doi.org/10.1152/japplphysiol.00798.2003

Antoniades C., Shirodaria C., Leeson P., Antonopoulos A., Warrick N., Van-Assche T., Cunnington C., Tousoulis D., Pillai R., Ratnatunga C., Stefanadis C., Channon K. M. (2009): Association of plasma asymmetrical dimethylarginine (ADMA) with elevated vascular superoxide production and endothelial nitric oxide synthase uncoupling: implications for endothelial function in human atherosclerosis. Eur. Heart J. 30, $1142-1150$ http://dx.doi.org/10.1093/eurheartj/ehp061

Asemu G., Neckar J., Szarszoi O., Papousek F., Ostadal B., Kolar F. (2000): Effects of adaptation to intermittent high altitude hypoxia on ischemic ventricular arrhythmias in rats. Physiol Res. 49, 597-606

Barbier J., Reboul C., Goret L., Catheline M., Gibault., Dauzat M., Obert P., Tanguy S. (2007): Aortic vasoconstriction related to smooth muscle cells ET-A and ET-B receptors is not involved in hypoxia-induced sustained systemic arterial hypertension in rats. Vascular Pharmacol. 47, 209-214 http://dx.doi.org/10.1016/j.vph.2007.06.005

Barton C. H., Ni Z., Vaziri N. D. (2003): Blood pressure response to hypoxia: Role of nitric oxide syntase. Am. J. Hypertens. 16, 1043-1048 http://dx.doi.org/10.1016/j.amjhyper.2003.07.021

Bernardi L., Passino C., Serebrovskaya Z., Serebrovskaya T., Appenzeller O. (2001): Respiratory and cardiovascular adaptations to progressive hypoxia; effect of interval hypoxic training. Eur Heart J. 22, 879-886

http://dx.doi.org/10.1053/euhj.2000.2466

Barua R. S., Ambrose J. A., Eales-Reynolds L. J., DeVoe M. C., Zervas J. G., Saha D. C. (2001): Dysfunctional endothelial nitric oxide biosynthesis in healthy smokers with impaired endothelium-dependent vasodilatation. Circulation 104, 1905-1910 http://dx.doi.org/10.1161/hc4101.097525

Calbet J. A. (2003): Chronic hypoxia increases blood pressure and noradrenaline spillover in healthy humans. J. Physiol. 551, 379-386 http://dx.doi.org/10.1113/jphysiol.2003.045112
Cohen R. A., Weisbrod R. M. (1988): Endothelium inhibits norepinephrine release from adrenergic nerves of rabbit carotid artery. Am. J. Physiol. 254, 871-878

Dick T. E., Hsieh Y. H., Wang N., Prabhakar N. R. (2006): Acute intermittent hypoxia increases both phrenic and sympathetic nerve activities in the rat. Exp. Physiol. 92, 87-97 http://dx.doi.org/10.1113/expphysiol.2006.035758

Eid H. M., Eritsland. J, Larsen J., Arnesen H., Seljeflot I. (2003): Increased levels of asymmetric dimethylarginine in populations at risk for atherosclerotic disease. Effects of pravastatin. Atherosclerosis 166, 279-284 http://dx.doi.org/10.1016/S0021-9150(02)00206-X

Foster G. E., Poulin M. J., Hanly P. J. (2006): Intermittent hypoxia and vascular function Implications for obstructive sleep apnea. Exp. Physiol. 92, 51-65 http://dx.doi.org/10.1113/expphysiol.2006.035204

Greenberg S., Diecke F. P., Peevy K., Tanaka T. P. (1989): The endothelium modulates adrenergic neurotransmission to canine pulmonary arteries and veins. Eur. J. Pharmacol. 162, 67-80 http://dx.doi.org/10.1016/0014-2999(89)90605-5

Guner I., Yelmen N., Sahin G., Oruç T. (2002): The effect of intracerebroventricular dopamine administration on the respiratory response to hypoxia. Tohoku J. Exp. Med. 196, 219-230 http://dx.doi.org/10.1620/tjem.196.219

Guner I., Yelmen N., Sahin G., Oruc T., Sipahi S., Yaman M. O. (2007): Respiratory alterations due to chronic long-term intermittent hypobaric hypoxia in rabbits: importance of peripheral chemoreceptors. Arch. Med. Res. 38, 739-745 http://dx.doi.org/10.1016/j.arcmed.2007.04.010

Guner I., Sahin G., Yelmen N. K., Aksu U., Oruc T., Yildirim Z. (2008): Intracerebroventricular serotonin reduces the degree of acute hypoxic ventilatory depression in peripherally chemodenervated rabbits. Chin. J. Physiol. 51, 136-145

Hansen J., Sander M. (2003): Sympathetic neural overactivity in healthy humans after prolonged exposure to hypobaric hypoxia. J. Physiol. 546, 921-929 http://dx.doi.org/10.1113/jphysiol.2002.031765

Johnson T. S., Young J. B., Landberg L. (1983): Sympathoadrenal responses to acute and chronic hypoxia in the rat. J. Clin. Invest. 71, 1263-1272 http://dx.doi.org/10.1172/JCI110876

Kim Y. J., Park H. S., Lee H. Y., Ha E. H., Suh S. H. Oh S. K., Yoo H. S. (2006): Reduced L-arginine level and decreased placental eNOS activity in preeclampsia. Placenta 27, 438-444 http://dx.doi.org/10.1016/j.placenta.2005.04.011

Meerson F. Z., Ustinova E. E., Orlova E. H. (1987): Prevention and elimination of heart arrhythmias by adaptation to intermittent high altitude hypoxia. Clin. Cardiol. 10, 783-789 http://dx.doi.org/10.1002/clc.4960101202

Meerson F. Z., Ustinova E. E., Manukhina E. B. (1989): Prevention of cardiac arrhythmias by adaptation to hypoxia: Regulatory mechanisms and cardiotropic effect. Biomed. Biochim. Acta 38, S83-88

Meerson F. Z., Mashina S., Lapshin A. V., Manukhina E. B. (1993): Decrease in constrictor response and increase in dilator response of the resistance artery in experimental myocardial infarction: effect of adaptation to hypoxia on this phenomenon. Biull. Eksp. Biol. Med. 115, 464-466 (in Russian) 
Moncada S., Higgs E. A. (1995): Molecular mechanisms and therapeutic strategies related to nitric oxide. FASEB J. 9, 1319-1330

Neubauer J. A. (2001): Invited review: Physiological and patophysiological responses to intermittent hypoxia. J. Appl. Physiol. 90, 1593-1599

Ni Z., Bemanian S., Kivlighn S. D., Vaziri N. D. (1998): Role of endothelin and nitric oxide imbalance in the pathogenesis of hypoxia-induced arterial hypertension. Kidney Int. 54, 188-192 http://dx.doi.org/10.1046/j.1523-1755.1998.00987.x

Prabhakar N. R. (2001): Oxygen sensing during intermittent hypoxia: Cellular and molecular mechanisms. J. Appl. Physiol. 90, 1986-1994

Prabhakar N. R., Kumar G. K. (2004): Oxidative stress in the systemic and cellular responses to intermittent hypoxia. Biol. Chem. 385, 217-221 http://dx.doi.org/10.1515/BC.2004.015

Rodriguez F. A., Ventura J. L., Casas M., Casas H., Pages T., Rama R., Ricart A., Palacios L. Viscor G. (2000): Erythropoietin acute reaction and haematological adaptations to short, intermittent hypobaric hypoxia. Eur. J. Appl. Physiol. 82, 170-177 http://dx.doi.org/10.1007/s004210050669

Row B. W., Kheriandish L., Neville J. J., Gozal D. (2002): Impared spatial learning and hyperactivity in developing rats exposed to intermittent hypoxia. Pediatr. Res. 52, 449-453 http://dx.doi.org/10.1203/00006450-200209000-00024

Semenza G. L. (2000). HIF-1: mediator of physiological and pathophysiological responses to hypoxia. J. Appl. Physiol. 88, 1474-1480

Serebrovskaya T. V., Swanson J., Kolesnikova E. E. (2003): Intermittent hypoxia: Mechanisms of action and some applications to bronchial asthma treatment. J. Physiol. Pharmacol. 54, 35-41

Serebrovskaya T. V., Manukhina E. B., Smith M. L., Downey H. F., Mallet R. T. (2008): Intermittent Hypoxia: Cause or therapy for systemic hypertension? Exp. Biol. Med. 233, 627-650 http://dx.doi.org/10.3181/0710-MR-267

Surdacki A., Nowicki M., Sandmann J., Tsikas D., Boeger R. H., Bode-Boeger S. M., Kruszelnicka-Kwiatkowska O., Kokot F., Dubiel J. S., Froelich J. C. (1999): Reduced urinary excretion of nitric oxide metabolites and increased plasma levels of asymmetric dimethylarginine in men with essential hypertension. J. Cardiovasc. Pharmacol. 33, 652-658 http://dx.doi.org/10.1097/00005344-199904000-00020
Tamisier R., Anand A., Nieto L. M., Cunnington D., Weiss J. W. (2005): Arterial pressure and muscle sympathetic nevre activity are increased after two hours of sustained but not cyclic hypoxia in healthy humans. J. Appl. Physiol. 98, 343-349 http://dx.doi.org/10.1152/japplphysiol.00495.2004

Vaziri N. D., Wang Z. Q. (1996): Sustained systemic arterial hypertension induced by extended hypobaric hypoxia. Kidney Int. 49, 1457-1463 http://dx.doi.org/10.1038/ki.1996.205

West J. B. (2004): The physiological basis of high-altitude disease. Ann. Inter. Med. 141, 789-800 http://dx.doi.org/10.7326/0003-4819-141-10-20041116000010

Yamamoto R., Wada A., Asada Y., Nina H., Sumiyoshi A. (1993): Nomega -nitro-L-arginine, an inhibitor of nitric oxide synthesis, decreases noradrenaline outflow in rat isolated perfused mesenteric vasculature. Arch. Pharmacol. 347, 238-240 http://dx.doi.org/10.1007/BF00169274

Yelmen N. K., Sahin G., Oruç T., Hacibekiroğlu M. (2003): Hypoxic initiation of pulmonary hypertension is mediated by serotonin secretion from neuroepithelial bodies in chemodenervated dogs. Chin. J. Physiol. 46, 27-33

Yelmen N. K. (2004): The role of gama-aminobutiric acid and glutamate for hypoxic ventilator responses in anesthetized rabbits. Tohoku J. Exp. Med. 203, 219-232

http://dx.doi.org/10.1620/tjem.203.219

Yelmen N. K., Turgut G., Sahin G., Oruç T. (2004): Effects of intravenous and intracerebroventricular theophylline on hypoxic ventilatory depression in anesthetized cats. Med. Princ. Pract. 13, 277-281 http://dx.doi.org/10.1159/000079527

Yelmen N., Ozdemir S., Guner I., Toplan S., Sahin G., Yaman O. M., Sipahi S. (2011): The effects of chronic long-term intermittent hypobaric hypoxia on blood rheology parameters. Gen. Physiol. Biophys. 30, 389-395 http://dx.doi.org/10.4149/gpb_2011_04_389

Zhong N., Zhang Y., Zhu H. F., Wang J. C., Fang Q. Z., Zhou Z. N. (2002): Myocardial capillary angiogenesis and coronary flow in ischemia tolerance rat by adaptation to intermittent high altitude hypoxia. Acta Pharmacol. Sin. 23, 305-310

Received: February 20, 2013

Final version accepted: September 10, 2013 